\title{
Critical Casimir Effect in Three-Dimensional Ising Systems: Measurements on Binary Wetting Films
}

\author{
Masafumi Fukuto, ${ }^{*}$ Yohko F. Yano, ${ }^{\dagger}$ and Peter S. Pershan \\ Department of Physics and Division of Engineering and Applied Sciences, Harvard University, \\ Cambridge, Massachusetts 02138, USA
}

(Received 3 January 2005; published 8 April 2005)

\begin{abstract}
The critical Casimir force (CF) is observed in thin wetting films of a binary liquid mixture close to the liquid/vapor coexistence. X-ray reflectivity shows thickness $(L)$ enhancement near the bulk consolute point. The extracted Casimir amplitude $\Delta_{+-}=3 \pm 1$ agrees with the theoretical universal value for the antisymmetric 3D Ising films. The onset of CF in the one-phase region occurs at $L / \xi \sim 5$ regardless of whether the bulk correlation length $\xi$ is varied with temperature or composition. The shape of the Casimir scaling function depends monotonically on the dimensionality.
\end{abstract}

An important unresolved issue of critical phenomena relates to the prediction by Fisher and de Gennes [1] of a critical fluctuation-induced Casimir force $(\mathrm{CF})$ between the outer surfaces of a fluid near its bulk critical point (BCP) $[2,3]$. The $\mathrm{CF}$ in quantum electrodynamics originates from confinement of zero-point fluctuations of the vacuum fields between conducting plates [4,5]. The analogous thermodynamic $\mathrm{CF}$ arises from the effect of confining a fluid with diverging bulk correlation length $\xi$ to a finite dimension $L$. Although the underlying theoretical understanding of this "critical" CF is relatively well established $[2,3]$, experimental tests of the theory have begun to appear only recently. These studies are typically carried out on thin wetting films of near-critical fluids because in the planar geometry the $\mathrm{CF}$ contributes directly to the force balance that determines the equilibrium film thickness $[2,3,6]$. For example, recent studies of superfluid ${ }^{4} \mathrm{He}$ and ${ }^{4} \mathrm{He} /{ }^{3} \mathrm{He}$ mixture films $[7,8]$ support the predictions for critical CF in $X Y$ systems. In the first experiments on the more ubiquitous Ising systems, Mukhopadhyay and Law [9] observed enhancement in the thickness of binary hydrocarbon mixture films near the demixing BCP. However, while their results qualitatively follow theoretical expectations, the extracted strength of CF turned out to be more than 2 orders of magnitude smaller than predicted for the 3D Ising films. This discrepancy may be due to the use of partial or incomplete wetting films, for which the theoretical assumption of the finite-size scaling limit is not well satisfied $[3,10]$. In view of the absence of other experimental evidence, a quantitative understanding of the phenomenon has yet to be firmly established.

We present an independent measurement of the critical $\mathrm{CF}$ in the 3D Ising film that is formed by complete wetting of a $\mathrm{SiO}_{2} / \mathrm{Si}$ substrate by a binary liquid mixture of methylcyclohexane $\left[(\mathrm{MC}) \mathrm{C}_{6} \mathrm{H}_{11}-\mathrm{CH}_{3}\right]$ and perfluoromethylcyclohexane $\left[(\mathrm{PF}) \mathrm{C}_{6} \mathrm{~F}_{11}-\mathrm{CF}_{3}\right]$ [11]. The demixing $\mathrm{BCP}$ is at $T_{c}=46.2{ }^{\circ} \mathrm{C}$ and PF mole fraction of $x=x_{c} \approx 0.36$ [12]. A previous study [13] demonstrated that at $x \sim x_{c}$, this mixture completely wets the $\mathrm{SiO}_{2} / \mathrm{Si}$ surface at $30{ }^{\circ} \mathrm{C}$ with antisymmetric boundary conditions (BCs), where $\mathrm{MC}$ wets the $\mathrm{SiO}_{2} /$ film interface and $\mathrm{PF}$ wets the film/vapor interface. In the present study, we measured film thickness variations about the $\mathrm{BCP}$ and extracted the dependence of the Casimir pressure scaling function on both $T$ and $x$. For the thick films that form close to the bulk liquid/vapor coexistence, the measured magnitude of $\mathrm{CF}$ agrees well with the theoretical universal amplitude.

The theoretical predictions for critical CF [2,3,14-19] assert that in the finite-size scaling limit $T \rightarrow T_{c}, \xi \rightarrow \infty$, and $L \rightarrow \infty$, the critical contribution to the free energy per unit area for a film of critical composition and thickness $L$ in $d$-dimensional space has the form $\omega_{c}(T, L) \sim$ $k_{B} T_{c} L^{-(d-1)} \Theta(L / \xi)$. The corresponding Casimir pressure is $P_{c}(T, L)=-\partial \omega_{c}(T, L) / \partial L \sim k_{B} T_{c} L^{-d} \vartheta(L / \xi)$, where $\vartheta(z)=(d-1) \Theta(z)-z \Theta^{\prime}(z)$ [6]. The universal scaling functions $\Theta$ and $\vartheta$ are close to zero for $L / \xi \gg 1$ but increase as $L / \xi \rightarrow 1$ and reduce to the so-called universal "Casimir amplitude," $\Delta \equiv \Theta(0)=\vartheta(0) /(d-1)$, exactly at the $\mathrm{BCP}$ or $L / \xi=0$. For an Ising film with antisymmetric BCs [denoted as $(+-)$ ], the order parameters $\phi$ at the two opposing surfaces are finite and have opposite signs at all $T[14,15,20]$. Functional forms of $\Theta_{+-}$or $\vartheta_{+-}$are yet to be determined for $d=3$; however, both the exact calculation at $d=2$ [14] and mean-field calculation [15], corresponding to $d=4$, suggest that $\vartheta_{+-}(L / \xi)$ should be positive and peaked slightly below bulk $T_{c}$ [see Fig. 3(d)]. The amplitude $\Delta_{+-}$has been estimated for $d=3$ Ising films: $\Delta_{+-}=2.39$ from a renormalization group (RG) theory [15], 2.45 from a Monte Carlo simulation [15], and 3.1 from the "local free energy functional" (LFEF) theory [16].

The three experimentally controlled variables are represented in Fig. 1: (i) the reduced temperature $t=$ $\left(T-T_{c}\right) / T_{c}$ of the film, (ii) the reduced mole fraction $\phi=$ $x-x_{c}$ in the bulk liquid reservoir, and (iii) the offset $\Delta \mu>0$ by which the intrinsic chemical potential of the liquid film deviates from the bulk liquid/vapor coexistence. In this work, $\Delta \mu$ is varied through the temperature differ- 


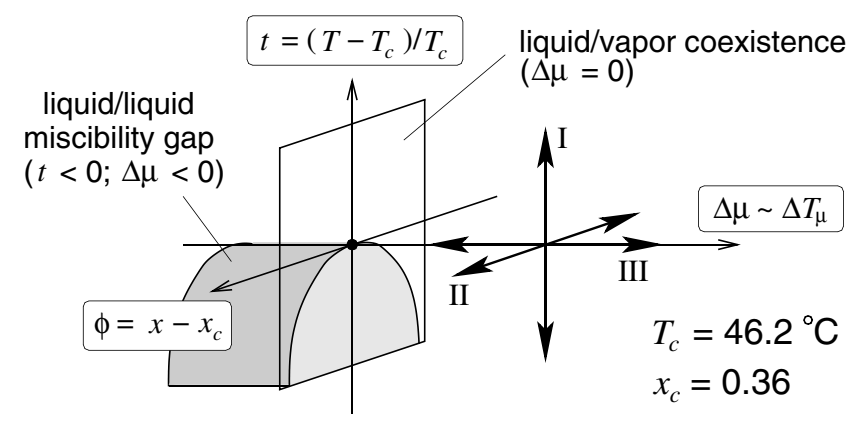

FIG. 1. Schematic phase diagram consisting of three experimental variables. Wetting films are formed on the $\Delta \mu>0$ side, where vapor is the stable bulk phase. Film thickness data was collected along paths I, II, and III for different $\Delta \mu$.

ence $\Delta T_{\mu}>0$ between the film and the reservoir, where $\Delta \mu \propto \Delta T_{\mu}$ for small $\Delta T_{\mu}[13,21]$. For noncritical dielectric liquids, the thickness of a complete wetting film is determined by the balance between $\Delta \mu$ and the van der Waals (vdW) interactions with the substrate $[22,23]$. For a critical fluid, the CF also contributes to this balance. Assuming a nonretarded vdW potential $\omega_{\mathrm{vdW}}=W / L^{2}[24]$, the equilibrium thickness $L$ can be expressed as [6]

$$
L=\left[\left\{2 W+k_{B} T_{c} \vartheta(L / \xi)\right\} / \Delta \mu\right]^{1 / 3},
$$

where $W=A_{\text {eff }} / 12 \pi$ ( $>0$ for wetting films) is the effective Hamaker constant between the two-component film and the substrate, and $\Delta \mu$ is defined as per unit volume. Since $L \rightarrow \infty$ as $\Delta \mu \rightarrow 0^{+}$, approaching the origin $(t, \phi, \Delta \mu)=(0,0,0)$ corresponds to the finite-size scaling limit, for which universal behavior is predicted $[2,3,14,17-$ 19]. Given that $W$ is expected to depend only weakly on $T$ and monotonically on $x$, any near-critical enhancement in $L$ at fixed $\Delta \mu$, e.g., along paths I and II in Fig. 1, is a direct measure of $\vartheta_{+-}(L / \xi)>0$. Measurement along path III, i.e., as a function of $\Delta \mu$ at $(t, \phi)=(0,0)$, is suited for probing $\Delta_{+-}$, which is expected to converge to a universal value as $\Delta \mu \rightarrow 0^{+}$.

The cell and cleaning procedure for the Si substrate [11] were described previously [13]. Differential heating of the substrate [13,21] stabilizes films up to $L \sim 150 \AA$ [or $\Delta T_{\mu} \sim 0.02 \mathrm{~K}$ for $\mathrm{MC} / \mathrm{PF}$ at $\left.\left(T_{c}, x_{c}\right)\right]$. The estimated uncertainty in $\Delta T_{\mu}$ is $\pm 3 \mathrm{mK}$. A liquid reservoir of target $x$ was formed by injecting appropriate amounts of $\mathrm{MC}$ and $\mathrm{PF}$ [11] that accounted for the saturated vapor [12]. The reservoir was stirred for $3 \mathrm{~h}$ at $60{ }^{\circ} \mathrm{C}$ prior to the measurements starting at or above $T_{c}$ and at $30^{\circ} \mathrm{C}$ for those starting below $T_{c}$. For x-ray reflectivity [25], the $\mathrm{Cu} K \alpha_{1}$ radiation $(\lambda=1.540 \AA)$ of a fixed anode tube was reflected off of a vertically oriented substrate in the horizontal scattering plane [13] at an incident angle $\alpha$, corresponding to wave vector transfer $q_{z}=(4 \pi / \lambda) \sin (\alpha)$ normal to the surface. Each scan was taken to $q_{z} \sim 0.3 \AA^{-1}$ (reflectivity $R \sim$ $10^{-6}$ ) over $4-5 \mathrm{~h}$. An accurate, model-independent mea- sure of the total film thickness $L=\left\langle n \pi / q_{z, n}\right\rangle$ was obtained from the extremum positions $q_{z, n}$ ( $>0.1 \AA^{-1}$ ) of the welldefined interference ("Kiessig") fringes arising from the substrate/film and film/vapor interfaces [13]. At given $\left(T, \Delta T_{\mu}\right)$, scans were repeated over $0.5-1.5$ days to ensure that thickness was stable to within $1 \AA$ for $>12 \mathrm{~h}$.

Measured $L$ at selected $\Delta T_{\mu}$ offsets is plotted as a function of $T$ at $x_{c}$ in Fig. 2(a) (along path I in Fig. 1) and of $x$ at $T_{c}$ in Fig. 2(b) (path II). The film thickening near $\left(T_{c}, x_{c}\right)$ that is evident for small $\Delta T_{\mu}(\leq 0.1 \mathrm{~K})$ signifies the presence of critical CF. At $x_{c}$, the maximum $L$ at each $\Delta T_{\mu}[=0.1$ and $0.02 \mathrm{~K}$ in Fig. 2(a) $]$ is obtained slightly below $T_{c}$. The thickening is observed regardless of the direction of temperature variation. However, for $T<$ $T_{c}$ (the two-phase coexistence regime in bulk), the measured $L$ displays a hysteresis between cooling and heating $\left[\Delta T_{\mu}=0.1 \mathrm{~K}\right.$ in Fig. 2(a)]; the two data sets thus provide upper and lower limits on the true equilibrium thickness. In contrast, for $T>T_{c}$ (the single-phase regime in bulk), hysteresis is not observed and the data should closely reflect the true equilibrium. As for the $x$ dependence at $T_{c}$ [Fig. 2(b)], the maximum $L$ is obtained when $x \sim x_{c}$ for the reservoir, suggesting that the composition in the film is also close to criticality. This and the relatively flat top of the $L$ enhancement imply that the data at $x_{c}$ can be used with confidence to extract both $\Delta_{+-}$and $\vartheta_{+_{-}}$for a mean-

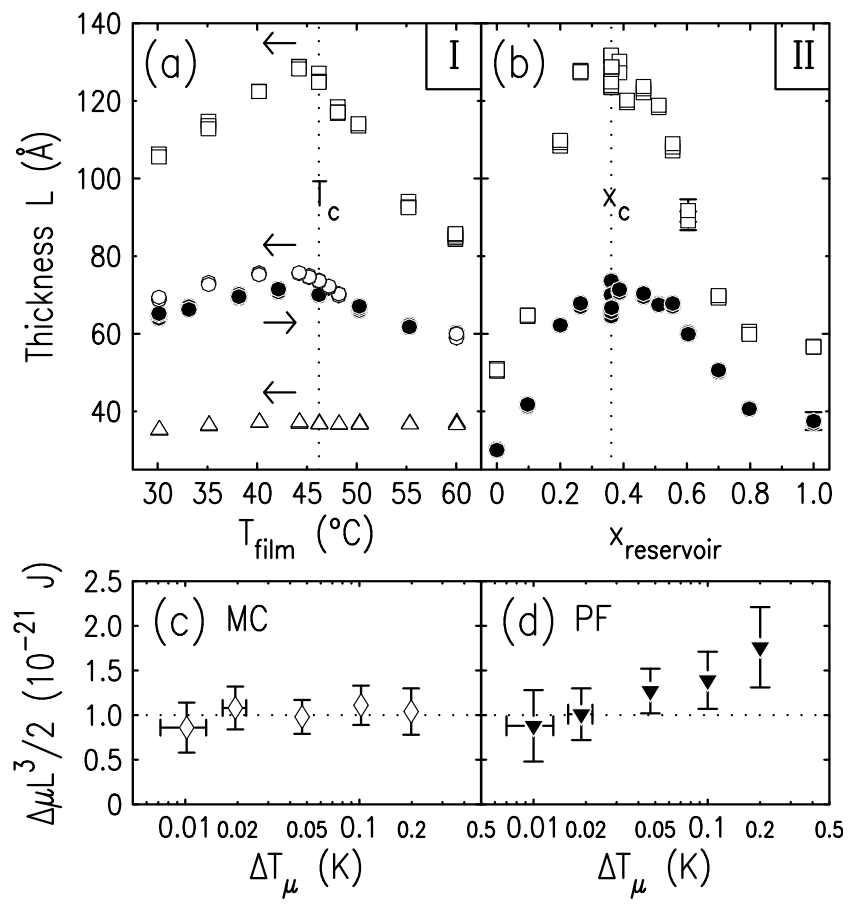

FIG. 2. (a) Measured thickness $L$ vs $T$, at $x_{c}$ for $\Delta T_{\mu}=$ $0.02 \mathrm{~K}$ (squares), $0.1 \mathrm{~K}$ (circles), and $0.5 \mathrm{~K}$ (triangles). Open and closed symbols represent data on cooling $(\leftarrow)$ and heating $\left(\rightarrow\right.$ ), respectively. (b) $L$ vs $x$, at $T_{c}$ for $\Delta T_{\mu}=0.02 \mathrm{~K}$ (squares) and $0.1 \mathrm{~K}$ (circles). Measured $W=\Delta \mu L^{3} / 2$ vs $\Delta T_{\mu}$ for (c) pure $\mathrm{MC}$ and (d) pure $\mathrm{PF}$ on $\mathrm{SiO}_{2} / \mathrm{Si}$ at $46.2{ }^{\circ} \mathrm{C}$. 
ingful comparison with the existing calculations, all of which implicitly assume a film of critical composition.

Extraction of $\vartheta_{+-}$requires an estimate of $W(12 \mid S)$ for the mixture films, where the indices 1,2 , and $S$ denote $\mathrm{PF}, \mathrm{MC}$, and the $\mathrm{SiO}_{2} / \mathrm{Si}$ substrate, respectively. For a uniform single-phase mixture film, one can show $W(12 \mid S) \approx\left(n_{1} / n_{1}^{\circ}\right) W(1 \mid S)+\left(n_{2} / n_{2}^{\circ}\right) W(2 \mid S)+$ $\left(n_{1} / n_{1}^{\circ}\right)\left(n_{2} / n_{2}^{\circ}\right) \epsilon_{12}$, where $n_{i}$ and $n_{i}^{\circ}$ are the number density of each component in the mixture and pure films, respectively. With Hamaker constants $W_{i j}=A_{i j} / 12 \pi$ for interactions between wetting molecules, $\epsilon_{12} \equiv W_{11}+$ $W_{22}-2 W_{12} \approx 0.048 \times 10^{-21} \mathrm{~J}$ [13]. Figures $2(\mathrm{c})$ and 2(d) show measured $W(i \mid S)=\Delta \mu L^{3} / 2$ for pure films, where $\Delta \mu=\Delta \mu_{i}^{\circ}=n_{i}^{\circ}\left(Q_{i} \Delta T_{\mu} / T+m_{i} g H\right)$ [13,21], $Q_{i}$ is the latent heat, $m_{i}$ is the molar mass, and $H=40 \mathrm{~mm}$ for the height of the $\mathrm{x}$-ray spot above the reservoir. Whereas the assumption of nonretarded vdW interactions can be justified for pure MC on Si from the near constancy of $\Delta \mu L^{3} / 2$, PF exhibits a deviation from the expected power law $L \sim \Delta \mu^{-1 / 3}$ at large $\Delta T_{\mu}$ (i.e., small $L$ ), possibly due to the presence of additional short-range interactions. Nevertheless, the data for the most relevant region of small $\Delta T_{\mu}$ (large $L$ ) show $W(1 \mid S) \sim W(2 \mid S) \sim 1 \times 10^{-21} \mathrm{~J} \gg$ $\epsilon_{12}$. Noting $n_{1} / n_{1}^{\circ}+n_{2} / n_{2}^{\circ}=1$ in the absence of the volume of mixing, we conservatively estimate $W(12 \mid S)=$ $(1.0 \pm 0.5) \times 10^{-21} \mathrm{~J}$. Neglecting the entropies of mixing for both liquid and vapor $(<1 \%$ of the entropy of vaporization $Q_{i} / T$ near the BCP $), \Delta \mu=\left(n_{1} / n_{1}^{\circ}\right) \Delta \mu_{1}^{\circ}+$ $\left(n_{2} / n_{2}^{\circ}\right) \Delta \mu_{2}^{\circ}$ for the binary films.

Figure 3 summarizes the experimentally derived $\vartheta_{+-}(L / \xi)$ based on the inverse of Eq. (1). The ratio $L / \xi$ was obtained using $\xi=\xi_{0}^{ \pm}|t|^{-\nu}$ for $T_{<}^{>} T_{c}$ at $x_{c}$ [Fig. 3(a)] and $\xi=l_{0}\left|\left(x-x_{c}\right) / x_{c}\right|^{-\nu / \beta}$ at $T_{c}$ [Fig. 3(b)], where $\nu=$ $0.63, \beta=0.32$, and $\xi_{0}^{+} / \xi_{0}^{-}=1.96$ for the 3D Ising systems [26]. For MC/PF, the bare correlation lengths are given by $\xi_{0}^{+}=2.8 \AA$ [27], and $l_{0}=6.4 \AA$, based on the relation $l_{0} \approx 0.38 \xi_{0}^{+}\left(B / x_{c}\right)^{\nu / \beta}$ for $d=3$ [28] and $B \approx$ 0.90 for the bulk MC/PF miscibility gap $\left|x-x_{c}\right|=B|t|^{\beta}$ at $T \lesssim T_{c}$ [12]. In Fig. 3(d), the $T$-dependent shape $\vartheta_{+-}(y) / \vartheta_{+-}(0)$ at $x_{c}$ is compared with the existing theories for $2 \mathrm{D}$ and $4 \mathrm{D}$ (mean-field) Ising films [14,15], where $y \equiv\left(L / \xi^{+}\right)^{1 / \nu}$ scales with $t$.

The presence of critical CF is clearly evidenced by the fact that the scaled data $\vartheta_{+-}$are always positive, exhibit a peak at $x \sim x_{c}$ and $T \lesssim T_{c}$, and fall off as $L / \xi \rightarrow \infty$. Another predicted property of $\vartheta_{+-}$is its universality in the critical regime, which implies that for sufficiently small $\Delta \mu$, the scaled data at different $\Delta \mu$ (or $\Delta T_{\mu}$ ) should collapse onto a single curve [2,3]. Evidence of this behavior can be seen in both the $T$ - and $x$-dependent data [Figs. 3(a) and 3(b)], which show a reasonable agreement between $\vartheta_{+-}$obtained at $\Delta T_{\mu}=0.02$ and $0.1 \mathrm{~K}$, despite the difference in $L$ by a factor of nearly 2 . By contrast, the offset of $\Delta T_{\mu}=0.5 \mathrm{~K}$, for which $\vartheta_{+-}$is considerably reduced [Figs. 3(a) and 3(c)], is clearly too large to be in the critical regime. Therefore, in the following, we restrict the direct comparison with theoretical calculations to the scaled data below $\Delta T_{\mu} \sim 0.1 \mathrm{~K}$.

The most significant result of this study is that the data at $\left(T_{c}, x_{c}\right)$ and the smallest experimentally achievable offset of $\Delta T_{\mu}=0.02 \mathrm{~K} \quad$ [Figs. 3(a) $-3(\mathrm{c})$ ] yield $\Delta_{+-}=$ $\vartheta_{+-}(0) / 2=3 \pm 1$ for the Casimir amplitude of the antisymmetric 3D Ising films. This estimate, based on complete wetting films, agrees very well with the theoretical range of $\Delta_{+-}=2.4-3.1[15,16]$, in sharp contrast to the value $\Delta_{+-}=0.005-0.006$ extracted previously from partial wetting films [9]. The results here demonstrate that the magnitude of critical CF for complete wetting films is accurately described by the existing theories. The comparison between $\vartheta_{+_{-}}(0) / 2$ and $W / k_{B} T_{c}=0.23 \pm 0.11 \mathrm{im}-$ plies that at the $\mathrm{BCP}$, the Casimir pressure becomes roughly 1 order of magnitude greater than the vdW disjoining pressure. It is therefore highly unlikely that further
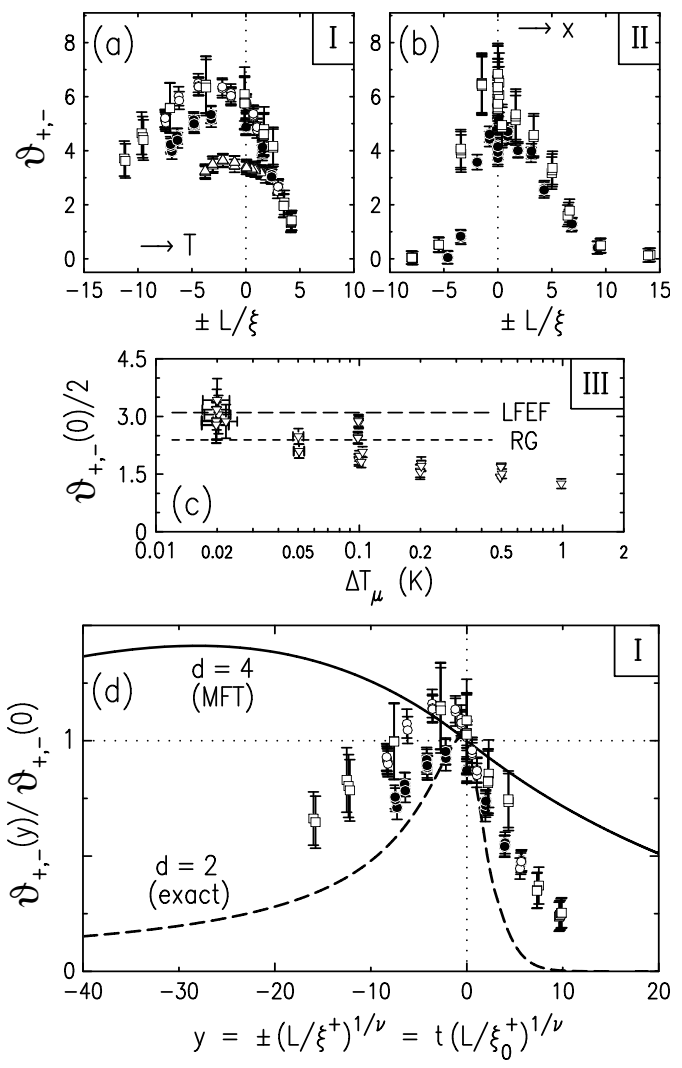

FIG. 3. The extracted Casimir pressure scaling function $\vartheta_{+-}$. (a) $T$ dependence at $x_{c}$, where $L / \xi_{<}^{\geq 0}$ for $T_{<}^{>} T_{c}$. (b) $x$ dependence at $T_{c}$, where $L / \xi_{<}^{>0}$ for $x_{<}^{>} x_{c}$. (c) $\vartheta_{+-}(0) / 2$ vs $\Delta T_{\mu}$ at $\left(T_{c}, x_{c}\right)$. The dashed lines indicate the theoretical universal amplitude for 3D Ising films: $\Delta_{+-}=2.39$ from RG theory [15] and 3.1 from LFEF theory [16]. (d) Comparison of $\vartheta_{+-}(y) / \vartheta_{+-}(0)$ vs $y=t\left(L / \xi_{0}^{+}\right)^{1 / \nu}$ between the measurement (3D data) and theory for 2D (dashed line; exact [14]) and 4D (line; mean-field [15]) Ising films. Roman numerals in (a)-(d) represent the paths in Fig. 1. Symbols in (a), (b), and (d) match those in Figs. 2(a) and 2(b). 
improvement in the estimate of $W$ would significantly alter the agreement between the theoretical and empirical $\Delta_{+--}$.

The scaled data provide an additional insight into the effect of dimensionality. While previous theoretical studies point toward greater magnitudes of $\vartheta_{+-}$at higher $d$ [1416,20], little attention has been paid to the effect of $d$ on the shape of $\boldsymbol{\vartheta}_{+-}$. As shown in Fig. 3(d), $\vartheta_{+-}(y)$ displays a qualitatively similar $t$-dependent behavior regardless of $d$. More quantitatively, however, it can be seen that the normalized data $\vartheta_{+-}(y) / \vartheta_{+-}(0)$ obtained for the $d=3$ Ising films display a shape that is intermediate between those predicted for $d=2$ and $d=4$. Specifically, the width $\Delta y$ of the Casimir enhancement region, the peak distance $\left|y_{\max }\right|$, and the relative peak height $\vartheta_{+-}\left(y_{\max }\right) / \vartheta_{+-}(0)$ all increase monotonically with $d$. While the origin of this behavior is not immediately clear, the result implies that theoretical evaluation of $\vartheta_{+-}$for the $d=3$ Ising films should satisfy this dimensional dependence.

The present work is unique in its examination of the compositional dependence of $\vartheta_{+-}$. Calculation of $\vartheta_{+-}$ along the $\phi$ axis is currently not available, preventing a direct comparison with this aspect of theory. Nevertheless, given that at $T_{c}$ both sides of $x=x_{c}$ correspond to the onephase region in bulk, it seems reasonable that the peak shape of $\vartheta_{+-}(L / \xi)$ along the $x$ axis [Fig. 3(b)] is more symmetric about $L / \xi=0$ than that along the $T$ axis [Fig. 3(a)]. Another significant observation is that in the single-phase regime, i.e., on the $L / \xi>0$ side of Fig. 3(a) and on both sides of $L / \xi=0$ in Fig. 3(b), the extent of the Casimir enhancement region $\vartheta_{+-}>0$ is limited roughly to $L / \xi \lesssim 5$. Thus, to a first approximation, the onset of the critical CF appears to be determined solely by the magnitude of $L / \xi$ and insensitive to whether $\xi$ is varied along $T$ or $x$. Further experimental studies are clearly needed to substantiate this rather exclusive dependence of the extent of $\vartheta_{+-}>0$ on the magnitude of $L / \xi$, as well as to find out whether it can be generalized to the two-phase region. On the other hand, the inference is consistent with the finitesize scaling hypothesis $[17,18]$, which asserts that for given universality class and geometry, $L / \xi$ is the only relevant parameter that dictates the crossover between bulk and finite-size scaling behaviors.

In summary, we have observed the critical Casimir effect in complete wetting films of binary hydro/fluorocarbon mixture. The estimate $\Delta_{+-}=3 \pm 1$ based on films near the liquid/vapor coexistence agrees well with the theoretical values of the universal Casimir amplitude for the antisymmetric 3D Ising films. The shape of the extracted Casimir scaling function $\vartheta_{+-}$is intermediate between the universal behaviors calculated previously for the 2D and 4D Ising films. The results in the single-phase region of the bulk phase diagram highlight the exclusive role that the magnitude of $L / \xi$ plays in determining the onset of the Casimir force.

We are grateful to $\mathrm{S}$. Dietrich for suggesting this experiment and to S. Dietrich, M. Krech, M. Kardar, F. Schlesener, and A. Gambassi for helpful discussions and suggestions. This work was supported by Grant No. NSF-DMR-01-24936.

*Present address: Department of Physics, Brookhaven National Laboratory, Upton, NY 11973.

Electronic address: fukuto@bnl.gov

${ }^{\dagger}$ Present address: Department of Chemistry, Gakushuin University, Toshima Ku, Tokyo 1718588, Japan.

[1] M. E. Fisher and P. G. de Gennes, C. R. Acad. Sci. (Paris), Ser. B 287, 207 (1978).

[2] M. Krech, The Casimir Effect in Critical Systems (World Scientific, Singapore, 1994), and references therein.

[3] M. Krech, J. Phys. Condens. Matter 11, R391 (1999).

[4] L. Spruch, Science 272, 1452 (1996).

[5] S. K. Lamoreaux, Am. J. Phys. 67, 850 (1999).

[6] M. Krech and S. Dietrich, Phys. Rev. A 46, 1922 (1992).

[7] R. Garcia and M.H.W. Chan, Phys. Rev. Lett. 83, 1187 (1999); 88, 086101 (2002).

[8] T. Ueno et al., Phys. Rev. Lett. 90, 116102 (2003).

[9] A. Mukhopadhyay and B. M. Law, Phys. Rev. Lett. 83, 772 (1999); Phys. Rev. E 62, 5201 (2000); 63, 041605 (2001).

[10] S. Dietrich and M. Krech (private communication).

[11] MC (Sigma-Aldrich, $99+\%$ ) and PF (Fluka, 97\%) were used as received. The substrate $\left(35 \times 25 \times 1 \mathrm{~mm}^{3}\right)$ was a Si(100) wafer (UMICORE Semiconductor, $p$-type, 1-10 $\Omega \mathrm{cm}$, measured roughness of $3 \AA$ ).

[12] R. B. Heady and J. W. Cahn, J. Chem. Phys. 58, 896 (1973).

[13] R. K. Heilmann, M. Fukuto, and P.S. Pershan, Phys. Rev. B 63, 205405 (2001).

[14] R. Evans and J. Stecki, Phys. Rev. B 49, 8842 (1994).

[15] M. Krech, Phys. Rev. E 56, 1642 (1997).

[16] Z. Borjan and P. J. Upton, Phys. Rev. Lett. 81, 4911 (1998).

[17] M.N. Barber, in Phase Transitions and Critical Phenomena, edited by C. Domb and J.L. Lebowitz (Academic, New York, 1983), Vol. 8, p. 145.

[18] V. Privman, in Finite Size Scaling and Numerical Simulation of Statistical Systems, edited by V. Privman (World Scientific, Singapore, 1990), p. 1.

[19] M. Krech and S. Dietrich, Phys. Rev. A 46, 1886 (1992).

[20] J. L. Cardy, Nucl. Phys. B275, 200 (1986).

[21] I. M. Tidswell, et al., Phys. Rev. Lett. 66, 2108 (1991).

[22] S. Dietrich, in Phase Transitions and Critical Phenomena, edited by C. Domb and J. L. Lebowitz (Academic, New York, 1988), Vol. 12, p. 1.

[23] B. M. Law, Prog. Surf. Sci. 66, 159 (2001).

[24] J. Israelachvili, Intermolecular and Surface Forces (Academic, London, 1991).

[25] J. Als-Nielsen and D. McMorrow, Elements of Modern $X$-Ray Physics (Wiley, Chichester, 2001).

[26] V. Privman, P. C. Hohenberg, and A. Aharony, in Phase Transitions and Critical Phenomena, edited by C. Domb and J. L. Lebowitz (Academic, New York, 1991), Vol. 14, p. 1.

[27] J. W. Schmidt, Phys. Rev. A 41, 885 (1990).

[28] F. Schlesener, A. Hanke, and S. Dietrich, J. Stat. Phys. 110, 981 (2003). 